\title{
An Economic Evaluation of Pembrolizumab Versus Other Adjuvant Treatment Strategies for Resected High-Risk Stage III Melanoma in the USA
}

\author{
Arielle G. Bensimon ${ }^{1} \cdot$ Zheng-Yi Zhou $^{2} \cdot$ Madeline Jenkins $^{2} \cdot$ Yan Song $^{1} \cdot$ Wei Gao ${ }^{1}$ James Signorovitch ${ }^{1}$.

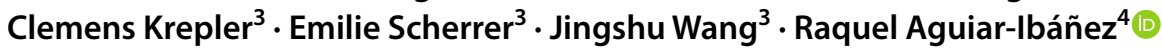

Published online: 16 May 2020

(c) The Author(s) 2020

\begin{abstract}
Background and Objective Over the past 5 years, adjuvant treatment options for surgically resected stage III melanoma have expanded with the introduction of several novel immune checkpoint inhibitors and targeted therapies. Pembrolizumab, a programmed cell death protein 1 inhibitor, received US Food and Drug Administration approval in 2019 for resected highrisk stage III melanoma based on significantly longer recurrence-free survival versus placebo. This study evaluated the cost-effectiveness of pembrolizumab versus other adjuvant treatment strategies for resected high-risk stage III melanoma from a US health system perspective.

Methods A Markov cohort-level model with four states (recurrence-free, locoregional recurrence, distant metastases, death) estimated costs and quality-adjusted life-years (QALYs) for pembrolizumab versus routine observation and other adjuvant comparators: ipilimumab in the overall population; and dabrafenib + trametinib in the BRAF-mutation positive (BRAF+) subgroup. Transition probabilities starting from recurrence-free were estimated through parametric multi-state modeling based on phase 3 KEYNOTE-054 (NCT02362594) trial data for pembrolizumab and observation, and network meta-analyses for other comparators. Post-recurrence transitions were modeled based on electronic medical records data and trials in advanced/metastatic melanoma. Utilities were derived using quality-of-life data from KEYNOTE-054 and literature. Costs of treatment, adverse events, disease management, and terminal care were included.

Results Over a lifetime, pembrolizumab, ipilimumab, and observation were associated with QALYs of 9.24, 7.09, and 5.95 and total costs of $\$ 511,290, \$ 992,721$, and $\$ 461,422$, respectively (2019 US dollars). Pembrolizumab was thus dominant (less costly, more effective) versus ipilimumab, with an incremental cost-effectiveness ratio of $\$ 15,155 /$ QALY versus observation. In the BRAF+ subgroup, pembrolizumab dominated dabrafenib + trametinib and observation, decreasing costs by $\$ 62,776$ and $\$ 11,250$ and increasing QALYs by 0.93 and 3.10 versus these comparators, respectively. Results were robust in deterministic and probabilistic sensitivity analyses.

Conclusions As adjuvant treatment for resected stage III melanoma, pembrolizumab was found to be dominant and therefore cost-effective compared with the active comparators ipilimumab and dabrafenib + trametinib. Pembrolizumab increased costs relative to observation in the overall population, with sufficient incremental benefit to be considered cost-effective based on typical willingness-to-pay thresholds.
\end{abstract}

\footnotetext{
Electronic supplementary material The online version of this article (https://doi.org/10.1007/s40261-020-00922-6) contains supplementary material, which is available to authorized users.

Raquel Aguiar-Ibáñez

raquel.aguiar-ibanez@merck.com

Analysis Group, Inc., Boston, MA, USA

Analysis Group, Inc., London, UK

Merck \& Co., Inc., Kenilworth, NJ, USA

4 Merck Sharp \& Dohme BV, Harleem, The Netherlands
} 


\section{Key Points}

Pembrolizumab was estimated to reduce costs and extend quality-adjusted life-years (QALYs) compared with active comparators for the adjuvant treatment of completely resected stage III melanoma, dominating ipilimumab in the overall population and dabrafenib + trametinib in the BRAF mutation-positive subgroup.

Pembrolizumab increased costs relative to the strategy of routine observation in the overall population, with sufficiently higher QALYs to be considered cost-effective from a US health system perspective.

\section{Introduction}

Melanoma is a type of skin cancer that develops from specialized pigmented cells known as melanocytes. In the USA, an estimated 91,270 new cases of melanoma were diagnosed in 2018, with 9320 associated deaths [1]. In the USA, 84\% of patients with melanoma are initially diagnosed at stage I-II (localized), 9\% at stage III (loco-regional), and $4 \%$ at stage IV (distant metastases), with 5-year survival ranging from $98 \%$ for stage I to $23 \%$ for stage IV melanoma [2]. Although most patients are diagnosed with localized disease and are cured, $20-30 \%$ of patients with early-stage melanoma later develop recurrence with higher rates observed in patients with regionally advanced disease [3].

The standard of care for resectable stage III disease is surgical excision with safety margins based on the presence and depth of invasion plus lymphadenectomy if regional lymph nodes are involved, followed by observation alone or with adjuvant therapy $[4,5]$. Systemic adjuvant therapy is recommended for patients with a high post-operative recurrence risk based on factors including tumor site, tumor thickness, ulceration, tumor mitotic count, or lymph node involvement [5-7]. Adjuvant therapy reduces the risk of recurrence and mortality by targeting residual micrometastatic disease [8].

The first adjuvant treatments to receive US Food and Drug Administration (FDA) approval were high-dose interferon$\alpha 2 b$ and peginterferon- $\alpha 2 b$, which showed modest efficacy and considerable toxicity [9-12]. Interferon-based regimens have since been displaced by the introduction of immune checkpoint inhibitors (anti-lymphocyte antigen-4 [CTLA4] and anti-programmed death-1 [PD-1] monoclonal antibodies) and targeted drugs (BRAF and MEK inhibitors, which are active exclusively against BRAF-mutated melanoma)
[13]. Adjuvant high-dose ipilimumab, a CTLA4 inhibitor, was FDA-approved in 2015 based on the European Organization for Research and Treatment of Cancer (EORTC) 18071 trial. At 5 years, recurrence-free survival (RFS) was $40.8 \%$ with ipilimumab versus $30.3 \%$ with placebo, with overall survival (OS) of $65.4 \%$ versus $54.4 \%$ [14, 15]. The PD-1 checkpoint inhibitor nivolumab was approved in 2017 for resected stage III/IV melanoma based on improvement in RFS versus ipilimumab in the CheckMate-238 trial (hazard ratio $[\mathrm{HR}]=0.65, p<0.0001)[16,17]$. For resected stage III BRAF mutation-positive (BRAF+) melanoma, dabrafenib + trametinib combination therapy was approved in 2018 based on improvements in RFS versus placebo in the COMBI-AD trial $(\mathrm{HR}=0.47, p<0.0001)[18,19]$.

Pembrolizumab is the latest adjuvant therapy to be FDAapproved (15 February 2019) for the adjuvant treatment of completely resected melanoma with lymph node involvement [20]. Pembrolizumab, an immune checkpoint inhibitor, is a highly selective monoclonal antibody that binds and blocks the PD-1 receptor of lymphocytes thereby reestablishing anti-tumor immunity by reactivating the tumorspecific cytotoxic T-lymphocytes that destroy tumor cells. FDA approval was based on results of EORTC-1325/KEYNOTE-054, a randomized, double-blind, placebo-controlled trial of 1,019 patients with completely resected, stage IIIA (> $1 \mathrm{~mm}$ lymph node metastasis), IIIB, or IIIC melanoma. Over 15 months of median follow-up, patients randomized to pembrolizumab experienced fewer recurrences/deaths ( $26.3 \%$ vs. $42.8 \%$ with placebo; $\mathrm{HR}=0.57, p<0.001$ ) [21]. Secondary endpoints of distant metastases-free survival (DMFS) and OS will be evaluated in the second interim and final analyses of KEYNOTE-054.

Despite recent progress in the adjuvant treatment of highrisk surgically resected melanoma, there are currently no published economic evaluations comparing different novel therapies in this indication. This study aimed to evaluate cost-effectiveness of adjuvant pembrolizumab following complete resection of stage III melanoma versus other adjuvant strategies. Comparators included observation (based on direct comparative evidence from KEYNOTE-054 [21]) and other adjuvant therapies (based on indirect comparative evidence from a systematic literature review and network meta-analysis [NMA] [22]).

\section{Methods}

\subsection{Model Overview}

A decision-analytic model was implemented in Excel 2016 (Microsoft Corp., Redmond, WA, USA) to examine the cost-effectiveness of adjuvant treatments for resected stage III melanoma. The analysis adopted a lifetime horizon and 
1-week cycle length with half-cycle correction. Outcomes included life-years (LYs), quality-adjusted LYs (QALYs), costs, and incremental cost-effectiveness ratios (ICERs). Annual discounting by $3.0 \%$ was used for both costs and effects, and direct healthcare costs were included per a US health system perspective, as stipulated by the reference case of the Second Panel on Cost-Effectiveness in Health and Medicine [23]. Where applicable, cost inputs were inflationadjusted to 2019 US dollars (USD) using the medical care component of the Consumer Price Index [24].

\subsection{Target Populations}

The overall target population included adults with complete resection of high-risk stage IIIA (>1 mm), IIIB, or IIIC melanoma, consistent with enrollment criteria in KEYNOTE-054 and EORTC-18071. The BRAF+ subgroup was modelled separately to include comparisons with dabrafenib + trametinib.

\subsection{Intervention and Comparators}

Pembrolizumab was modelled based on the FDA-approved, trial-based dosing schedule of $200 \mathrm{mg}$ administered intravenously (IV) every 3 weeks for up to 1 year or 18 doses.

Other FDA-approved adjuvant treatments recommended by National Comprehensive Cancer Network (NCCN) guidelines for resected stage III melanoma include nivolumab, dabrafenib + trametinib (BRAF+ subgroup), ipilimumab, and observation [5]. As of 2019, the guideline-recommended usage of ipilimumab is limited to cases of nodal recurrence after exposure to anti-PD-1 therapy; ipilimumab was nevertheless considered a relevant comparator based on its licensing and continued usage in clinical practice. Comparators in the overall target population thus included ipilimumab $(10 \mathrm{mg} / \mathrm{kg} \mathrm{IV}$ every 3 weeks for four doses followed by every 12 weeks, up to 3 years) and the observation strategy. Dabrafenib + trametinib (dabrafenib $150 \mathrm{mg}$ orally twice daily and trametinib $2 \mathrm{mg}$ orally once daily, up to 1 year) was compared to pembrolizumab and observation in the BRAF+ subgroup.

Nivolumab was not evaluated as a comparator after the feasibility assessment concluded that a comparison via NMA was not feasible due to confounding differences between CheckMate 238 and other trials in the network, including differences in eligibility criteria and control arm protocol [22]. Specifically, ipilimumab was administered for up to 1 year in CheckMate 238 [16], compared with up to 3 years in EORTC 18071 [14, 15]. Additionally, CheckMate 238 enrolled patients with resected stage IIIB, IIIC, or IV melanoma [16], while EORTC 18071 [14, 15] and KEYNOTE-054 [21] enrolled patients with resected stage IIIA-IIIC melanoma and excluded those with stage
IV disease. Because cross-trial differences with respect to ipilimumab treatment duration and stage of melanoma could not be adjusted and would result in biased comparisons of RFS between nivolumab versus other adjuvant therapies, nivolumab was excluded from the prior NMA study [22] and cost-effectiveness model.

\subsection{Model Structure}

A Markov cohort structure was developed in accordance with methods guidance for state transition models [25]. The structure was previously applied in a submission to the National Institute for Health and Care Excellence (NICE) and in a published US-based economic evaluation focusing on the comparison of pembrolizumab versus observation $[26,27]$. In this new application, the model framework was expanded to evaluate a broader set of adjuvant comparators.

Health states included recurrence-free (RF), locoregional recurrence (LR), distant metastases (DM), and death (Fig. 1). Patients started in the RF state following surgical resection, with age and percentage female consistent with the KEYNOTE-054 population at baseline (overall: 54 years, $38.4 \%$; BRAF+: 52 years, $40.2 \%$ ). Adjuvant therapy affected patients' risks of transitioning directly from RF to LR, DM, or death, but was assumed to provide no continuing therapeutic effect after LR or DM. Risks of transitioning from LR to DM or death were thus assumed equivalent across adjuvant strategies. Following DM, patients were assumed to receive first- and second-line therapies for advanced melanoma. Risks of transitioning from DM to death depended on the efficacy of the first-line therapies received. The distribution of individuals across health states was used in conjunction with state-specific utilities and costs to estimate LYs, QALYs, and costs.

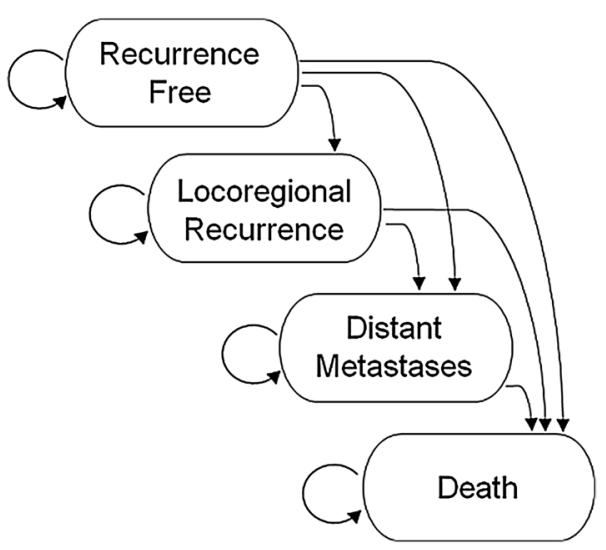

Fig. 1 Markov model schematic 


\subsection{Model Parameters}

\subsubsection{Efficacy}

A parametric multi-state modeling approach was used to estimate transition probabilities (TPs) between states [28-31]. Base-case parameter estimates are summarized in Table 1 (overall population) and the online supplement (BRAF+ subgroup). Background mortality rates served as a lower bound for all TPs to death [32].

Transitions starting from RF TPs from RF to other states were estimated using KEYNOTE-054 interim patientlevel data [21] and an NMA of adjuvant drug trials [22].
Parametric distributions were fitted in to the cause-specific hazards of each transition $(\mathrm{RF} \rightarrow \mathrm{LR}, \mathrm{RF} \rightarrow \mathrm{DM}$, and $\mathrm{RF} \rightarrow$ death) in the KEYNOTE-054 pembrolizumab and placebo arms, using R software (R Development Core Team, Vienna, Austria [33]). To account for competing risks, competing types of RFS failure were treated as censoring events [28-31]. For example, to model the $\mathrm{RF} \rightarrow \mathrm{DM}$ transition, patients with LR or death before DM were censored at the earlier competing event.

Six candidate distributions were considered to model transitions from $\mathrm{RF} \rightarrow \mathrm{LR}$ and $\mathrm{RF} \rightarrow \mathrm{DM}$, including exponential, Weibull, Gompertz, log-logistic, log-normal, and generalized gamma. Exponential distributions were used for $\mathrm{RF} \rightarrow$ death due to the small number of events [21]. All TPs

Table 1 Parametric models of health state transitions in the model

\begin{tabular}{|c|c|c|c|c|c|c|}
\hline \multirow[t]{2}{*}{ Transitions } & \multicolumn{3}{|c|}{ Parameter inputs (base case) } & \multirow[t]{2}{*}{ Data sources } & \multirow{2}{*}{$\begin{array}{l}\text { Scenario or one-way sensi- } \\
\text { tivity analyses performed }\end{array}$} & \multirow{2}{*}{$\begin{array}{l}\text { Distributions } \\
\text { used in probabil- } \\
\text { istic sensitivity } \\
\text { analyses }\end{array}$} \\
\hline & Pembrolizumab & Observation & Ipilimumab & & & \\
\hline $\mathrm{RF} \rightarrow \mathrm{LR}$ & $\begin{array}{l}\text { Gompertz } \\
\text { Shape }=-0.0252 \\
\text { Rate }=0.0040\end{array}$ & $\begin{array}{l}\text { Gompertz } \\
\text { Shape }=-0.0184 \\
\text { Rate }=0.0052\end{array}$ & $\begin{array}{l}\text { Parameters to } \\
\text { estimate time- } \\
\text { varying } \mathrm{HRs}^{\mathrm{b}} \text { : }\end{array}$ & $\begin{array}{l}\text { KEYNOTE-054 } \\
\text { data } \\
\text { US life tables }\end{array}$ & $\begin{array}{l}\text { Alternative parametric } \\
\text { distributions } \\
\text { Proportional hazards }\end{array}$ & $\begin{array}{l}\text { Exponential rate } \\
\text { of } \mathrm{RF} \rightarrow \text { death: } \\
\text { normal }\end{array}$ \\
\hline $\mathrm{RF} \rightarrow \mathrm{DM}$ & $\begin{array}{l}\text { Generalized gamma } \\
\text { Location }=6.5940 \\
\text { Scale }=2.1163 \\
\text { Shape }=0.3231\end{array}$ & $\begin{array}{l}\text { Generalized } \\
\text { gamma } \\
\text { Location }=4.9572 \\
\text { Scale }=1.6738 \\
\text { Shape }=-0.0704\end{array}$ & $\begin{array}{l}\mathrm{d} 0=-0.1494 \\
\mathrm{~d} 1=-0.07188\end{array}$ & $\begin{array}{l}\text { NMA of adju- } \\
\text { vant treatments } \\
\text { for melanoma }\end{array}$ & $\begin{array}{l}\text { parametric models with } \\
\text { a time-constant or time- } \\
\text { varying treatment effect }\end{array}$ & $\begin{array}{l}\text { All others: } \\
\text { multivariate } \\
\text { normal }\end{array}$ \\
\hline $\mathrm{RF} \rightarrow$ death $^{\mathrm{a}}$ & $\begin{array}{l}\text { Exponential } \\
\text { Rate }=0.00007\end{array}$ & $\begin{array}{l}\text { Exponential } \\
\text { Rate }=0.00004\end{array}$ & & & & \\
\hline $\mathrm{LR} \rightarrow \mathrm{DM}$ & $\begin{array}{l}\text { Exponential } \\
\text { Rate }=0.00874\end{array}$ & $\begin{array}{l}\text { Exponential } \\
\text { Rate }=0.00874\end{array}$ & $\begin{array}{l}\text { Exponential } \\
\text { Rate }=0.00874\end{array}$ & $\begin{array}{l}\text { Flatiron EHR } \\
\text { data }\end{array}$ & $\begin{array}{c}\text { Exponential rates of each } \\
\text { transition varied } \pm 10 \%\end{array}$ & Normal \\
\hline $\mathrm{LR} \rightarrow$ death $^{\mathrm{a}}$ & $\begin{array}{l}\text { Exponential } \\
\text { Rate }=0.00007\end{array}$ & $\begin{array}{l}\text { Exponential } \\
\text { Rate }=0.00007\end{array}$ & $\begin{array}{l}\text { Exponential } \\
\text { Rate }=0.00007\end{array}$ & $\begin{array}{l}\text { KEYNOTE-054 } \\
\text { data } \\
\text { US life tables }\end{array}$ & & Normal \\
\hline $\mathrm{DM} \rightarrow$ death $^{\mathrm{a}}$ & $\begin{array}{l}\text { Exponential } \\
\text { Rate }=0.0056 \text { if } \\
\text { entering DM } \\
\text { after } \geq 24 \text { months, } \\
\text { else } 0.0058\end{array}$ & $\begin{array}{l}\text { Exponential } \\
\text { Rate }=0.0054\end{array}$ & $\begin{array}{l}\text { Exponential } \\
\text { Rate }=0.0054\end{array}$ & $\begin{array}{l}\text { KEYNOTE-006 } \\
\text { data } \\
\text { NMA of treat- } \\
\text { ments for } \\
\text { advanced }\end{array}$ & $\begin{array}{l}\text { Alternative assumptions } \\
\text { about subsequent treat- } \\
\text { ments in each model arm } \\
\text { Exponential rates of OS } \\
\text { and PFS failure with }\end{array}$ & $\begin{array}{l}\text { Based on expo- } \\
\text { nential rates } \\
\text { of OS/PFS } \\
\text { failure with } \\
\text { treatments in }\end{array}$ \\
\hline $\begin{array}{l}\mathrm{DM} \rightarrow \text { post- } \\
\text { progression } \\
\mathrm{DM}^{\mathrm{c}}\end{array}$ & $\begin{array}{l}\text { Exponential } \\
\text { Rate }=0.0120 \text { if } \\
\text { entering DM } \\
\text { after } \geq 24 \text { months, } \\
\text { else } 0.0103\end{array}$ & $\begin{array}{l}\text { Exponential } \\
\text { Rate }=0.0111\end{array}$ & $\begin{array}{l}\text { Exponential } \\
\text { Rate }=0.0111\end{array}$ & $\begin{array}{l}\text { melanoma } \\
\text { Internal data } \\
\text { on the market } \\
\text { shares of first- } \\
\text { line treatments } \\
\text { for advanced } \\
\text { melanoma in } \\
\text { the US } \\
\text { US life tables }\end{array}$ & $\begin{array}{l}\text { treatments for advanced } \\
\text { melanoma varied } \pm 10 \%\end{array}$ & $\begin{array}{l}\text { the advanced } \\
\text { melanoma } \\
\text { setting } \\
\text { Pembrolizumab: } \\
\text { normal } \\
\text { All others: log- } \\
\text { normal }\end{array}$ \\
\hline
\end{tabular}

$D M$ distant metastases, $H R$ hazard ratio, $L R$ locoregional recurrence, $N M A$ network meta-analysis, $O S$ overall survival, $P F S$ progression-free survival, $R F$ recurrence-free

${ }^{a}$ Transition probabilities to death were constrained to be at least as high as all-cause mortality, as estimated from national life tables given the age and gender distribution of the cohort at each cycle

${ }^{\mathrm{b}}$ For ipilimumab, RF survival failure was modelled using time-varying HRs versus pembrolizumab, as estimated in a second-order fractional polynomial NMA of adjuvant treatments for melanoma

${ }^{\mathrm{c}}$ The exponential rate of disease progression in the DM state was used for the calculation of utility, disease management costs, and subsequent treatment costs in this state 
from RF depended upon all three cause-specific hazard functions. Base-case distributions were therefore selected from the 36 possible combinations of distributions for $\mathrm{RF} \rightarrow \mathrm{LR}$ and $\mathrm{RF} \rightarrow \mathrm{DM}$. In line with the approach recommended by the NICE Decision Support Unit (DSU) for partitioned survival models [34], the same base-case distribution types were selected for both treatment arms, with consideration of: goodness-of-fit between predicted versus observed RFS, and plausibility of long-term extrapolations based on external data [15]. As noted by the NICE DSU in separate guidance [30], assessing goodness-of-fit is more challenging in the context of multi-state models than partitioned survival models, as survival endpoints are determined by a combination of survival functions rather than by a single survival function [30]. Because Akaike Information Criterion and Bayesian Information Criterion do not provide suitable measures of fit in a multi-state model context, goodness-of-fit was instead assessed using mean squared error and the visual inspection approach described by Williams et al. [28]. Additional details are provided in the Online Supplemental Material.

Base-case distributions for $\mathrm{RF} \rightarrow \mathrm{LR}$ and $\mathrm{RF} \rightarrow \mathrm{DM}$ in the overall population were Gompertz and generalized gamma, respectively (Table 1). This combination was ranked first for placebo and fifth for pembrolizumab according to goodnessof-fit. Base-case distributions in the BRAF+ subgroup were Gompertz $(\mathrm{RF} \rightarrow \mathrm{LR})$ and log-normal $(\mathrm{RF} \rightarrow \mathrm{DM})$, ranked first for placebo and sixth for pembrolizumab. The Online Supplemental Material presents the overlay of predicted versus observed RFS for both treatment arms. Alternative distributions were tested in scenario analyses, summarized in Table 1 and detailed in the Online Supplemental Material.

For ipilimumab and dabrafenib + trametinib, causespecific hazards of transitioning from the RF state were derived using time-varying HRs of RFS for comparators versus pembrolizumab, estimated via second-order fractional polynomial NMA [22]. Within each weekly cycle, the HR was applied to the overall hazard of RFS failure under pembrolizumab. The proportion of the overall hazard attributable to each RFS failure type was assumed to be the same as in the pembrolizumab arm. To avoid clinically implausible long-term extrapolations, time-varying HRs for ipilimumab versus pembrolizumab were held constant after 70 weeks, corresponding to mean follow-up in KEYNOTE-054 at the first interim cutoff date. Due to the steeper upward trajectory of time-varying HRs for dabrafenib + trametinib versus pembrolizumab in the BRAF+ subgroup NMA, HRs for this comparator were conservatively held constant after 52 weeks. Time-constant HRs based on fixed-effects NMA were tested in scenario analyses.

Transitions starting from $L R$ In the absence of mature postrecurrence data from KEYNOTE-054, an exponential model of $\mathrm{LR} \rightarrow \mathrm{DM}$ was fitted using the Flatiron Health electronic medical record database (1/1/2011-2/28/2018) [35]. A realworld US cohort of 147 patients was followed from the date of LR after resection of stage III melanoma until DM or censoring. Because no direct transitions from $L R \rightarrow$ death were observed in the sample, the cause-specific hazard of $\mathrm{LR} \rightarrow$ death was assumed equal to that of RF $\rightarrow$ death estimated for pembrolizumab in KEYNOTE-054.

Transitions starting from DM For each adjuvant treatment, TPs from DM $\rightarrow$ death depended upon market shares of first-line treatments for advanced melanoma. The model also considered second-line treatment costs, but assumed that survival following DM would be driven by the choice of first-line therapy.

To represent real-world practice, market shares in the observation arm were obtained from unpublished US-specific market research (Table 2). The same market shares were assigned to patients in the ipilimumab arm, who were expected to be eligible for subsequent PD-1 inhibitors. In the pembrolizumab arm, patients' eligibility for subsequent PD-1 inhibitors was expected to depend on whether DM occurred early or late. Specifically, if DM occurred after $\geq 24$ months, $50.0 \%$ were assumed to receive rechallenge with pembrolizumab or nivolumab, while $50.0 \%$ receive other therapies; otherwise, patients were assumed to be ineligible for pembrolizumab retreatment, and were distributed to other subsequent treatments (Table 2).

Exponential OS and progression-free survival (PFS) distributions were estimated for each first-line subsequent treatment based on trials in the advanced melanoma setting. For pembrolizumab, these distributions were fitted using patientlevel data from the pembrolizumab $10 \mathrm{mg} / \mathrm{kg}$ every 3 weeks arm (first-line subgroup) of the KEYNOTE-006 trial among ipilimumab-naïve patients with unresectable/advanced melanoma [36]. For other first-line treatments, HRs for OS and PFS versus pembrolizumab were estimated through an NMA of first-line drug trials [36-43]. Expected OS following DM was calculated in each adjuvant treatment arm as a market share-weighted average of expected OS under different first-line treatments. Expected OS was then converted into a weekly hazard of DM $\rightarrow$ death. Expected PFS was estimated similarly for each adjuvant treatment arm.

\subsubsection{Safety}

Treatment-specific adverse event (AE) risks were considered for grade 3-5 drug-related AEs that affected $\geq 5.0 \%$ at any grade for pembrolizumab or placebo in KEYNOTE-054 [21], ipilimumab in EORTC-18071 [15], or dabrafenib + trametinib in COMBI-AD [18]. Diarrhea (grade $2+)$ was also considered based on the high expected cost of this AE. Mean durations for the AEs were collected from KEYNOTE-054. 


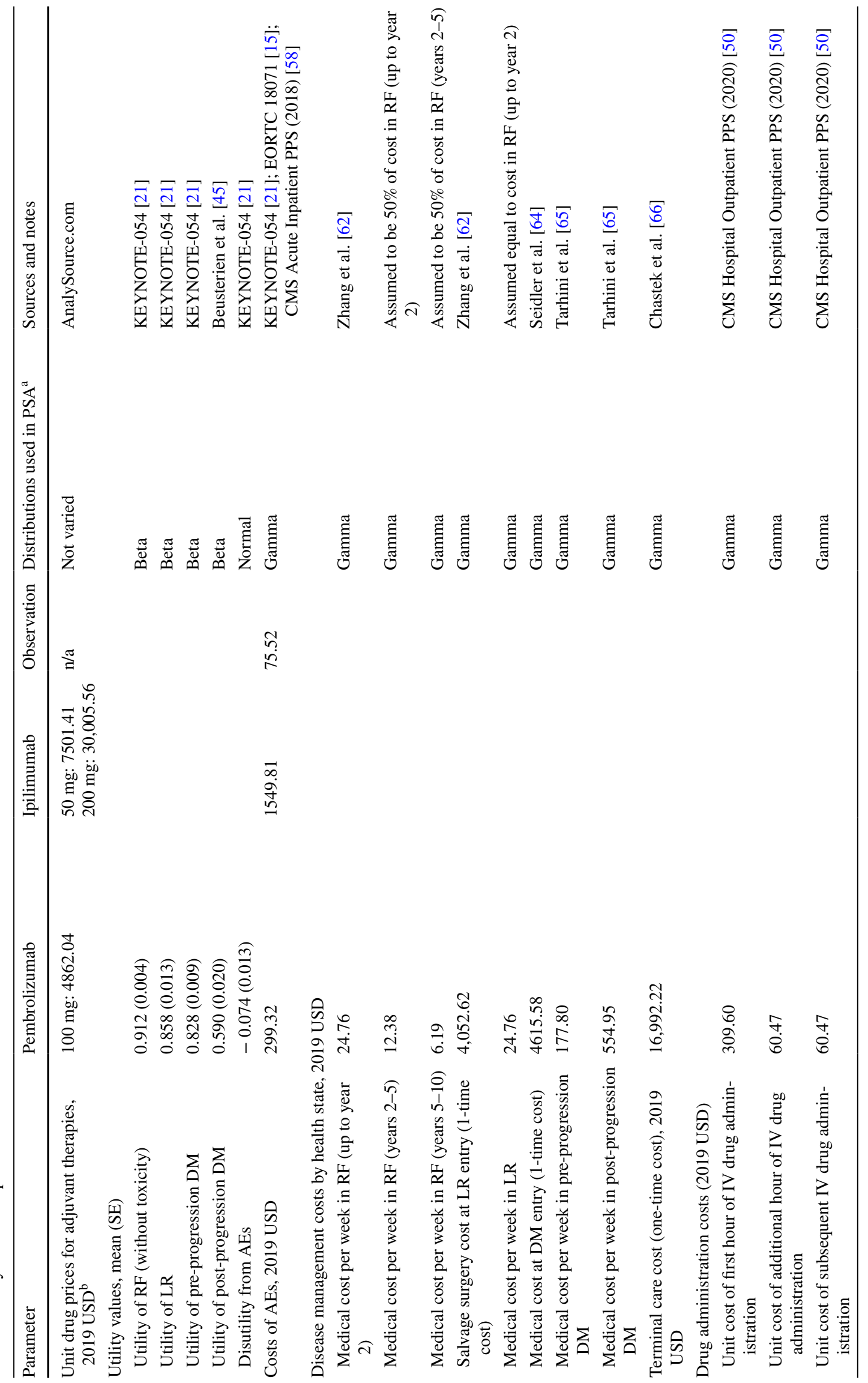




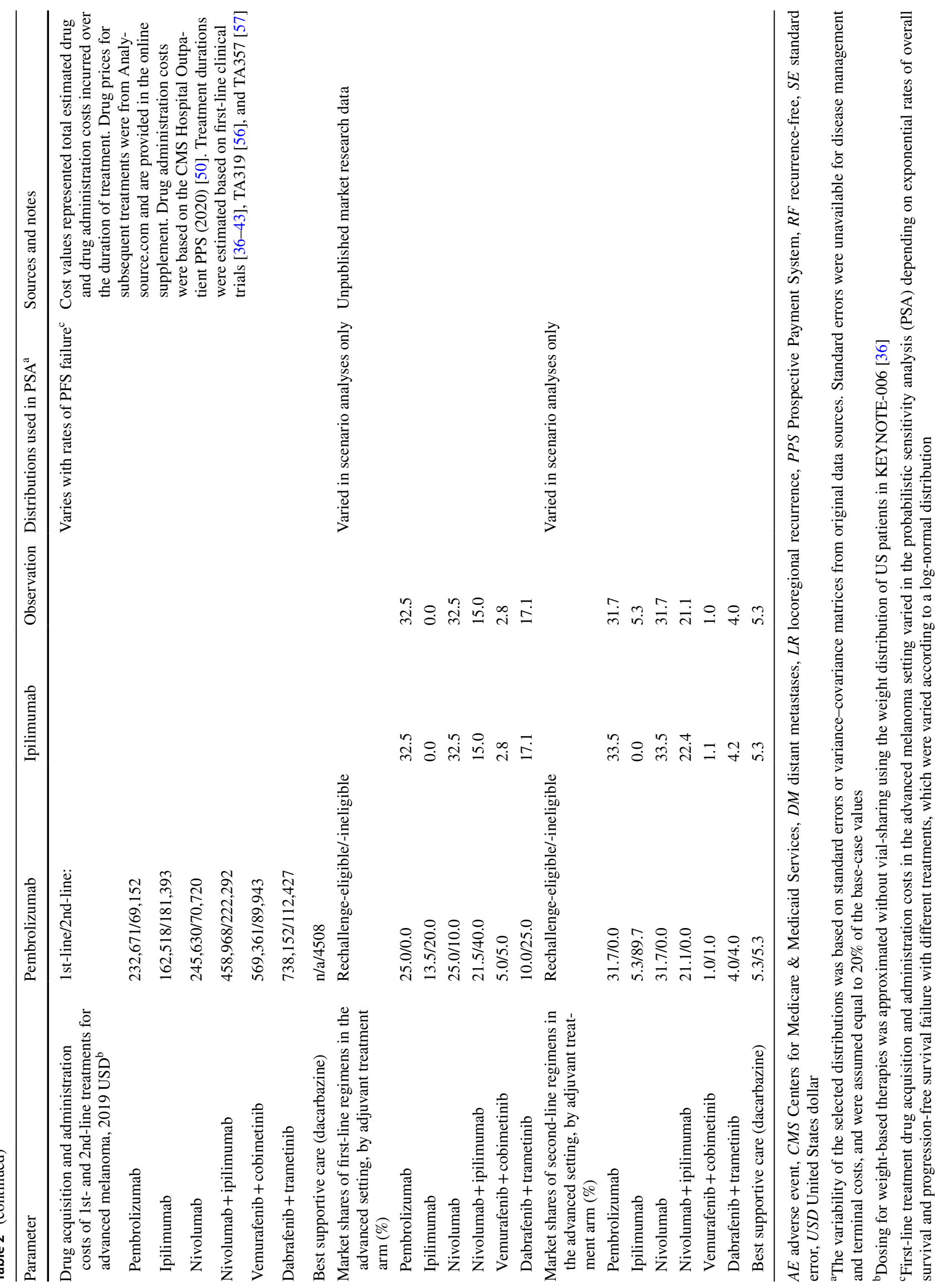




\subsubsection{Quality of life}

EuroQol-five dimension-three level (EQ-5D-3L) responses were collected during the KEYNOTE-054 trial and scored using the US value set [44]. Utilities for RF and LR were estimated through linear mixed-effects regression with patient-level random effects to link health states (defined according to RECIST V1.1 criteria) with EQ-5D-3L.

Utility in the DM state represented a weighted average of utilities associated with pre- and post-progression DM. The relative weight of these sub-state utilities depended on the estimated ratio of mean PFS:OS within the DM state, given the market shares of different first-line treatments. Utility for pre-progression DM was estimated based on EQ5D-3L data from KEYNOTE-054. Utility beyond progression could not be estimated reliably using KEYNOTE-054 data due to limited follow-up, and was therefore obtained from a cross-sectional study that elicited standard gamble utilities for advanced melanoma states [45]. Scenario analyses were conducted in which all utilities were informed by KEYNOTE-054, or by a different literature source [46].

AE-related disutility was estimated as the difference in utility associated with RF (without toxicity) versus RF (during any grade $3+\mathrm{AE}$ ) in KEYNOTE-054. For each adjuvant treatment, a one-time QALY decrement was applied in the first model cycle based on the risks, mean durations, and disutility of AEs.

\subsubsection{Costs}

Adjuvant drug costs were calculated based on their Wholesale Acquisition Cost (WAC) and label-recommended dosing $[15,18,21]$. In a scenario analysis, drug costs were instead calculated using Veterans Affairs national contract prices [47]. The relative dose intensity of pembrolizumab in KEYNOTE-054 (99.7\%) was also applied to drug acquisition costs in all adjuvant treatment arms to account for potential dose interruptions/reductions. IV administration costs were calculated based on minutes required per infusion (pembrolizumab: 30; ipilimumab: 90) and costs of IV administration services from the Centers for Medicare \& Medicaid Services (CMS) Hospital Outpatient Prospective Payment System [48-50] (Table 2). Orally administered drugs were assumed to require no administration costs.

For pembrolizumab, treatment duration was directly based on observed Kaplan-Meier data for time to discontinuation in KEYNOTE-054 (mean doses: 14), without need for extrapolation given the 1-year/18-dose maximum. For other adjuvant treatments, treatment duration was modeled using trial-based exponential distributions $[14,18]$, subject to FDA-recommended maximums (ipilimumab: 156 weeks; dabrafenib + trametinib: 52 weeks).
Costs of first- and second-line subsequent therapies were modeled as a lump-sum cost following DM (Table 2). The mean total cost of each regimen was calculated based on WAC, label-recommended dosing [48, 49, 51-55], drug administration costs [50], and estimated mean treatment duration. First-line treatment durations were modelled using the exponential PFS distributions, up to the label-recommended maximum where applicable. Second-line treatment durations were assumed to be 21 weeks (or the maximum duration if $<21$ weeks), in line with prior health technology appraisals in advanced melanoma [56, 57].

Unit costs of AEs were obtained from the CMS Acute Inpatient Prospective Payment System [58] and published sources [59-61]. These costs were multiplied by treatmentspecific AE risks and applied as a one-time cost in the first cycle.

Weekly medical cost in the RF state included outpatient services (e.g., physician office visits, radiologic assessments) reported in a retrospective study [62] (Table 2); this cost was assumed to decrease over time, based on guideline recommendations [5]. Following LR, a one-time cost of salvage surgery was applied based on the cost of surgery obtained from literature [62] and observed probabilities of lymphadenectomy, skin lesion resection, in-transit metastases resection, or other surgery after LR in KEYNOTE-054 [63]. Following DM, a one-time cost was applied based on medical resources associated with first-line treatment initiation [64]. Subsequent weekly medical costs were based on a retrospective chart review of US patients with unresectable stage III/ IV melanoma [65].

One-time costs of palliative/terminal care were applied for patients transitioning to death after DM [66].

\subsection{Sensitivity Analyses}

One-way deterministic sensitivity analyses (DSAs) and scenario analyses were conducted to examine the influence of specific inputs and assumptions. The Online Supplemental Material lists all DSAs performed in each target population.

Probabilistic sensitivity analysis (PSA) with 10,000 iterations was also conducted to estimate the probability of each treatment being cost-effective under different willingnessto-pay thresholds. In each iteration, inputs were randomly drawn from their specified distributions (Tables 1, 2). Standard errors or variance-covariance matrices of the selected distributions were based on original data sources, where available.

\subsection{Model Validation}

Model predictions for RFS, DMFS and OS are presented in Fig. 2. The model was internally and externally validated [67]. Clinical opinion was sought to validate efficacy 
inputs and other key model assumptions. Internal validity was assessed by comparing modeled efficacy against original sources that informed the efficacy inputs. RFS curves predicted for pembrolizumab and observation were plotted alongside observed Kaplan-Meier curves to assess their alignment. Model predictions for ipilimumab and dabrafenib + trametinib were similarly compared to reported statistics in the EORTC-18071 and COMBI-AD trials, respectively. To assess external validity, external data from the placebo arm of EORTC-18071 (median follow-up: 5.3 years) was used to validate 5-year OS projections for observation.

\section{Results}

\subsection{Base-Case Results}

In the overall population, total costs were $\$ 511,290$ for pembrolizumab, $\$ 461,422$ for observation, and $\$ 992,721$ for
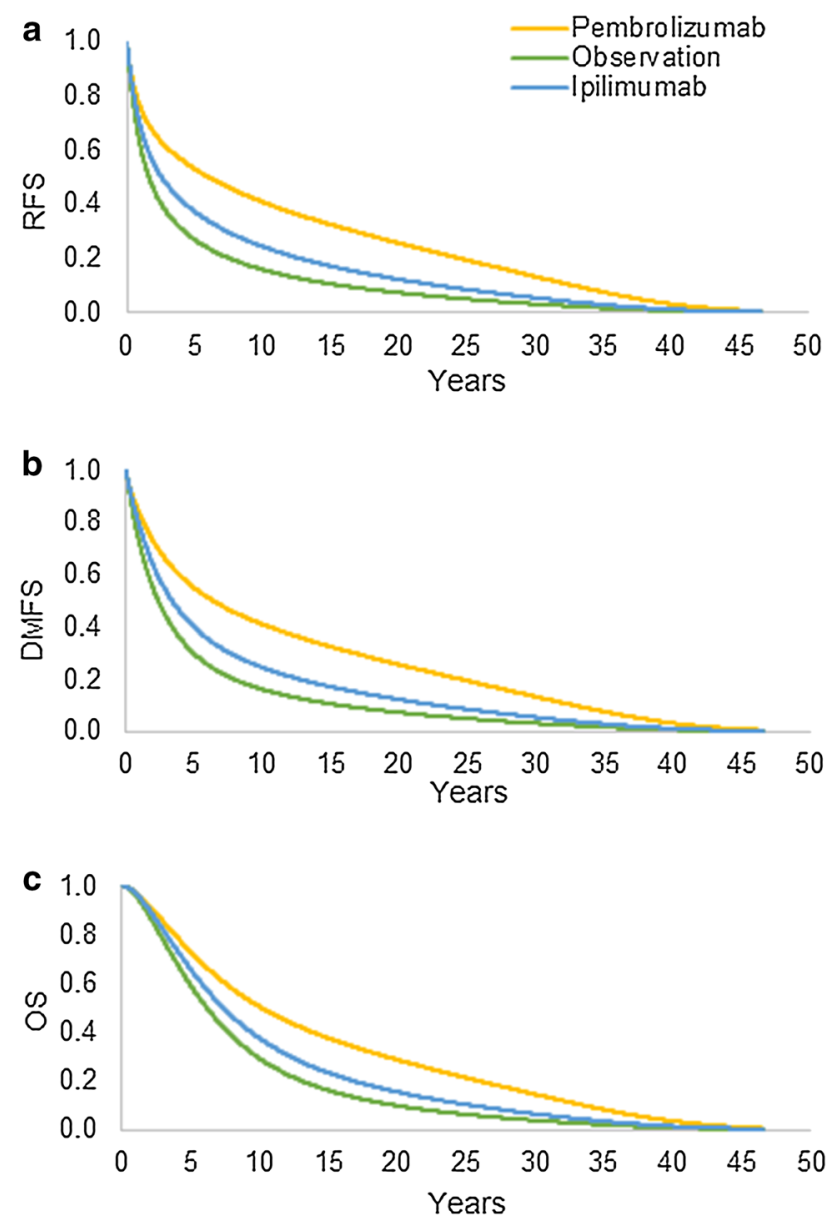

Fig. 2 Modelled long-term a RFS, b DMFS, and $\mathbf{c}$ OS in the overall population. DMFS distant metastases-free survival, $O S$ overall survival, $R F S$ recurrence-free survival ipilimumab (Table 3). Cost differences were largely attributable to adjuvant treatment costs, which were zero for observation and over fourfold higher for ipilimumab than pembrolizumab. Total QALYs were 9.24 for pembrolizumab, 5.95 for observation, and 7.09 for ipilimumab, with total LYs of $10.54,7.15$, and 8.35 , respectively. The proportion of LYs spent recurrence-free was $80.3 \%$ with pembrolizumab versus $57.3 \%$ with observation and $66.6 \%$ with ipilimumab. The resulting ICERs of pembrolizumab versus observation were $\$ 15,155 / \mathrm{QALY}$ and $\$ 14,691 / \mathrm{LY}$. Pembrolizumab dominated (i.e., was less costly and more effective than) ipilimumab.

In the BRAF+ subgroup, pembrolizumab dominated dabrafenib + trametinib, with incremental total costs of - \$62,776 and incremental QALYs and LYs of 0.93 and 0.91 , respectively. In this subgroup, pembrolizumab also dominated observation, with incremental total costs of - \$11,250 and incremental QALYs and LYs similar to those estimated in the overall population.

\subsection{Deterministic Sensitivity Analysis (DSA) and Scenario Analysis Results}

Tornado diagrams in Fig. 3 present the ten most influential sensitivity analyses in comparisons between pembrolizumab versus each comparator. Results are displayed in terms of ICERs or (for comparisons in which pembrolizumab was dominant in the base case) incremental net monetary benefits (INMB) using a \$100,000/QALY willingness-to-pay threshold.

Relative to observation, pembrolizumab ranged from dominant to having an ICER of $\$ 60,185 / \mathrm{QALY}$ in the overall population or $\$ 15,442 / \mathrm{QALY}$ in the BRAF+ subgroup. Pembrolizumab dominated ipilimumab in all sensitivity analyses and dominated dabrafenib + trametinib in all but two scenarios. The results were most sensitive to parameters determining RFS. Other influential parameters included time horizon, discount rate for effectiveness, RF-related utility, subsequent treatment assumptions, and use of Veterans Affairs national contract prices for drugs.

\subsection{Probabilistic Sensitivity Analysis (PSA) Results}

PSA results are illustrated through cost-effectiveness acceptability curves in Fig. 4. At a willingness-to-pay of $\$ 100,000 /$ QALY, the probability of cost-effectiveness in the overall population was $88.9 \%$ for pembrolizumab, $0.0 \%$ for ipilimumab, and $11.1 \%$ for observation. In the BRAF+ subgroup, the probability of cost-effectiveness was $78.6 \%$ for pembrolizumab, $17.7 \%$ for dabrafenib + trametinib, and 3.6\% for observation. 
Table 3 Base-case cost-effectiveness results by target population

\begin{tabular}{|c|c|c|c|c|c|c|}
\hline \multirow[t]{2}{*}{ Outcomes $^{\mathrm{a}}$} & \multicolumn{3}{|c|}{ Overall population } & \multicolumn{3}{|c|}{ BRAF+ subgroup } \\
\hline & Pembrolizumab & Ipilimumab & Observation & Pembrolizumab & $\begin{array}{l}\text { Dab- } \\
\text { rafenib + trametinib }\end{array}$ & Observation \\
\hline Total costs $(\$)$ & 511,290 & 992,721 & 461,422 & 520,812 & 583,588 & 532,062 \\
\hline Adjuvant treatment costs & 137,963 & 571,445 & 0 & 137,864 & 225,978 & 0 \\
\hline Drug acquisition costs & 135,801 & 569,926 & 0 & 135,704 & 225,978 & 0 \\
\hline Drug administration costs & 2162 & 1519 & 0 & 2160 & 0 & 0 \\
\hline Subsequent treatment costs & 322,859 & 353,633 & 389,815 & 325,939 & 289,368 & 463,938 \\
\hline Drug acquisition costs & 319,686 & 349,406 & 385,195 & 322,836 & 284,995 & 460,229 \\
\hline Drug administration costs & 3172 & 4226 & 4621 & 3104 & 4373 & 3709 \\
\hline Adverse event costs & 299 & 1,550 & 76 & 253 & 1020 & 81 \\
\hline Disease management costs & 40,960 & 54,503 & 58,857 & 46,996 & 56,347 & 55,227 \\
\hline Terminal care costs & 9209 & 11,590 & 12,674 & 9759 & 10,875 & 12,816 \\
\hline QALYs & 9.24 & 7.09 & 5.95 & 9.07 & 8.15 & 5.97 \\
\hline Recurrence-free & 7.71 & 5.07 & 3.73 & 7.46 & 6.40 & 3.73 \\
\hline Locoregional recurrence & 0.23 & 0.30 & 0.34 & 0.19 & 0.11 & 0.30 \\
\hline Distant metastases & 1.30 & 1.72 & 1.88 & 1.42 & 1.64 & 1.94 \\
\hline AE-related disutility & -0.0005 & -0.0018 & -0.0003 & -0.0005 & -0.0037 & -0.0004 \\
\hline Life-years & 10.54 & 8.35 & 7.15 & 10.43 & 9.52 & 7.12 \\
\hline Recurrence-free & 8.46 & 5.56 & 4.10 & 8.18 & 7.02 & 4.10 \\
\hline Locoregional recurrence & 0.27 & 0.35 & 0.39 & 0.23 & 0.12 & 0.35 \\
\hline Distant metastases & 1.81 & 2.43 & 2.66 & 2.03 & 2.38 & 2.68 \\
\hline \multicolumn{7}{|l|}{ ICERs } \\
\hline ICER (\$/QALY) & - & Dominant & 15,155 & - & Dominant & Dominant \\
\hline ICER (\$/life year) & - & Dominant & 14,691 & - & Dominant & Dominant \\
\hline
\end{tabular}

ICER incremental cost-effectiveness ratio, $Q A L Y$ quality-adjusted life-year

${ }^{a}$ Base-case results are reported with $3.0 \%$ annual discounting of costs and health benefits

\section{Discussion}

This study evaluated the cost-effectiveness of pembrolizumab, the latest FDA-approved adjuvant treatment option for resected stage III melanoma, compared with ipilimumab, dabrafenib + trametinib, and observation alone.

Results suggest that pembrolizumab is cost-effective relative to each comparator based on the common willingnessto-pay threshold of $\$ 100,000 / \mathrm{QALY}[68,69]$. In the overall population, the base-case ICER of pembrolizumab versus observation was $\$ 15,155 / \mathrm{Q} A L Y$. Pembrolizumab was dominant over ipilimumab in the base-case analysis, all PSA iterations, and across all input values and scenarios tested in DSAs. This finding was driven by ipilimumab leading to shorter RFS and higher adjuvant drug costs given the longer label-recommended maximum duration. Costs of subsequent treatments, disease management, and terminal care were also lower for pembrolizumab, reflecting the lower incidence of recurrence. In the context of a rapidly evolving treatment landscape in this indication, NCCN guidelines recommend adjuvant treatment with ipilimumab only in limited instances of nodal recurrence as of 2019 [5].
In the BRAF+ subgroup, pembrolizumab dominated dabrafenib + trametinib, and was estimated to be slightly cost-saving and therefore dominant over observation. Subsequent treatment costs were higher in the observation arm, the combined result of greater recurrence risk and larger expected market share of dabrafenib + trametinib (the most costly subsequent treatment).

Sensitivity analysis results were largely consistent with the base case, showing the most variation when using alternative distributions to model transitions starting from RF, which drive long-term survival extrapolations. In DSAs, the highest ICERs versus observation (overall: $\$ 60,185 /$ QALY; BRAF+: \$15,442/QALY) resulted from using parametric models with time-constant treatment effects; this alternative approach produced comparatively worse fit with observed RFS than the base-case parametric models individually fitted to each KEYNOTE-054 arm. Relative to dabrafenib + trametinib, INMB was negative only in two scenarios that used alternative distributions to model $\mathrm{RF} \rightarrow \mathrm{LR}$ and $\mathrm{RF} \rightarrow \mathrm{DM}$ transitions, which overestimated 3-year RFS in the dabrafenib + trametinib arm by $12-17$ percentagepoints relative to that observed in COMBI-AD (58\%) [18]. 


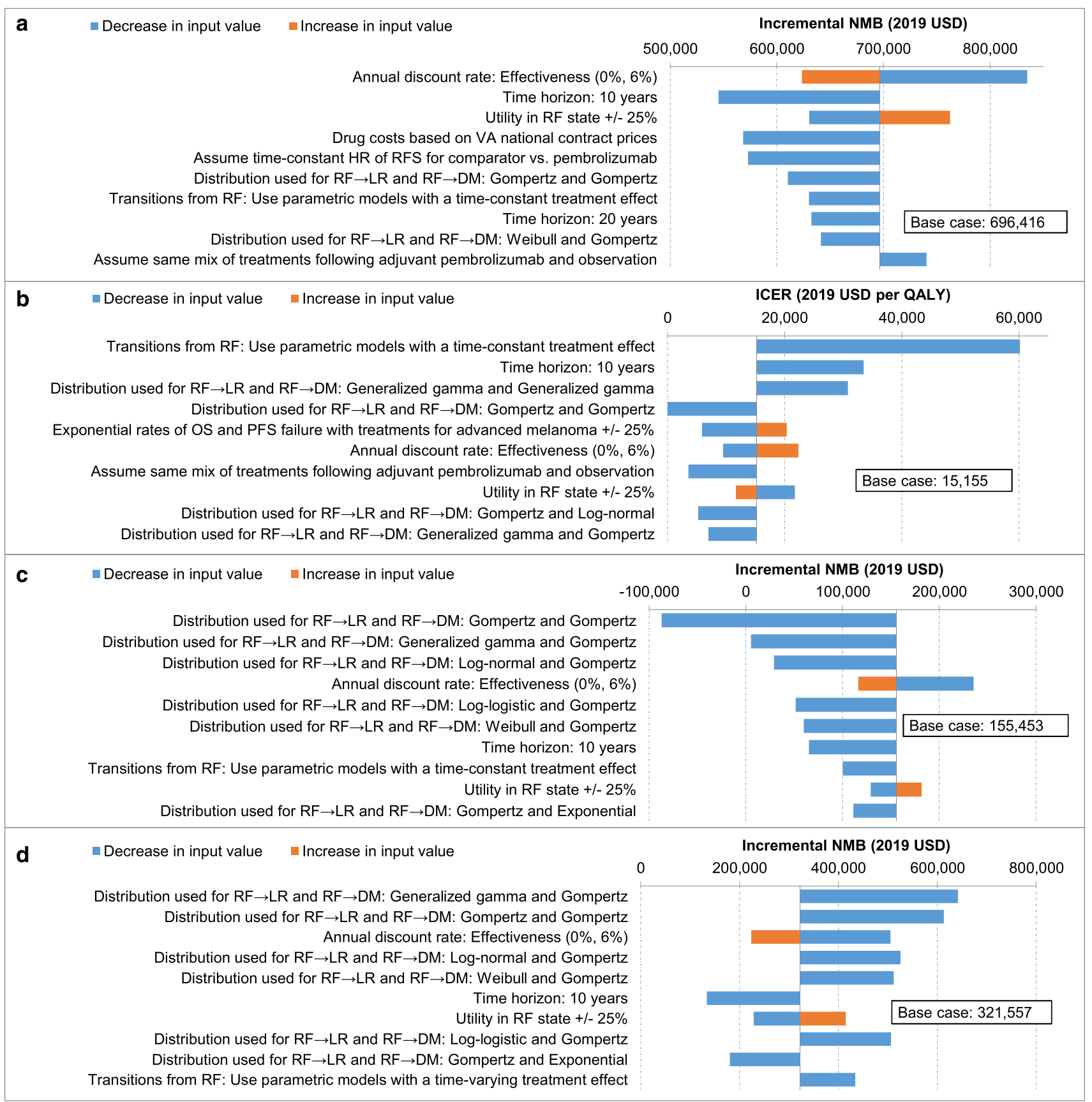

Fig. 3 Tornado diagrams based on deterministic sensitivity analysis (DSA) of pembrolizumab versus: a ipilimumab and $\mathbf{b}$ observation in the overall population; and $\mathbf{c}$ dabrafenib + trametinib and $\mathbf{d}$ observation in the BRAF+ subgroup. $D M$ distant metastases, $H R$ hazard

Patient-level data from KEYNOTE-054 were used to parametrically model transitions determining RFS for pembrolizumab and observation. Post-recurrence transitions were modeled based on real-world data and clinical trials of advanced melanoma treatments given the absence of longterm survival data from KEYNOTE-054. Extrapolation of OS using RFS is an approach supported by recent evidence ratio, ICER incremental cost-effectiveness ratio, $L R$ locoregional recurrence, $N M B$ net monetary benefit, $Q A L Y$ quality-adjusted lifeyear, $R F$ recurrence-free, $R F S$ recurrence-free survival, $U S D$ United States dollar, $V A$ Veterans Affairs on the natural history of high-risk melanoma after resection [70]. Additionally, in the EORTC-18071 trial, relative reductions in recurrence/death with ipilimumab versus placebo $(\mathrm{HR}=0.76)$ closely matched relative reductions in death $(\mathrm{HR}=0.72)$. Five-year OS in the placebo arm of EORTC18071 (54.4\%) was comparable to modeled OS in the observation arm $(59.0 \%)$ [15]. 


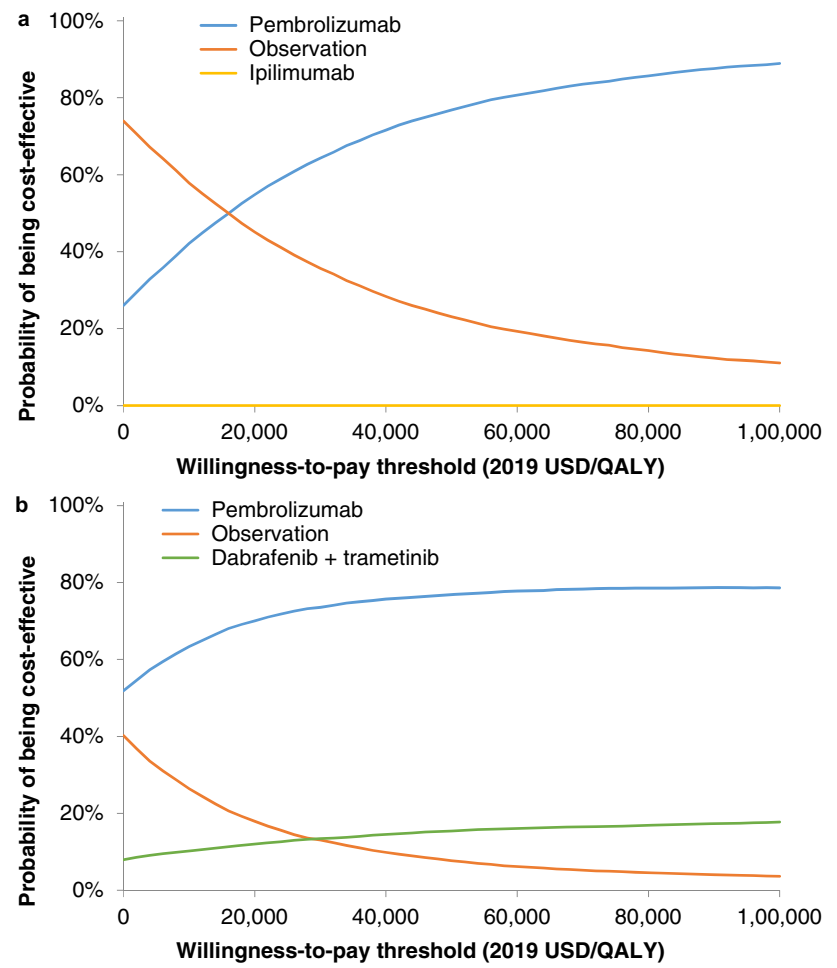

Fig. 4 Combined cost-effectiveness acceptability curves: a overall population; and b BRAF+ subgroup

With the recent FDA approval of several adjuvant treatments for resected stage III melanoma, comprehensive economic evaluations of these treatments are needed to inform reimbursement decision-making by payers. The cost-effectiveness of pembrolizumab was previously evaluated relative to routine observation in a US-based study [26]. Prior submissions to NICE have assessed the costeffectiveness of pembrolizumab [27], nivolumab [71], and dabrafenib + trametinib [72] from a UK national payer perspective, each focusing on the comparison with observation. The present study is the first published cost-effectiveness analysis to comparatively evaluate different active adjuvant treatments for melanoma.

Notable strengths of this study include modelling the RFS efficacy of pembrolizumab and observation based on headto-head trial data. Consistent with best practice, selection of parametric functions was based on goodness-of-fit with observed data and clinical plausibility of long-term projections [30]. Duration of pembrolizumab treatment was precisely estimated using observed data, without extrapolation. Utility values for most health states were directly measurable in KEYNOTE-054 based on EQ-5D-3L, a generic preference-based measure recommended by the Second Panel on Cost-Effectiveness in Health and Medicine [23].

This study is nevertheless subject to limitations. First, there are no direct head-to-head trials comparing pembrolizumab to comparators other than observation. A systematic literature review and NMA were conducted to indirectly compare RFS across adjuvant therapies [22]. Time-varying HRs from a fractional polynomial NMA were used to account for non-proportionality of hazards; scenario analyses using time-constant HRs yielded similar results.

Second, due to between-trial heterogeneity in treatment protocols and inclusion criteria, nivolumab could not be evaluated in the NMA [22] or cost-effectiveness model. Patients randomized to ipilimumab received treatment for $\leq 1$ year in CheckMate-238, compared with $\leq 3$ years in EORTC-18071. CheckMate-238 also enrolled patients with resected stage IIIB-C/IV disease, while EORTC-18071 and KEYNOTE-054 enrolled those with resected stage IIIA-C disease. Consequently, ipilimumab outcomes are not comparable across trials, preventing balanced indirect comparisons of nivolumab versus other adjuvant comparators; a similar limitation was noted by the Evidence Review Group that evaluated the NICE submission for nivolumab [73].

Third, DMFS and OS data were not part of the pre-specified interim analyses of KEYNOTE-054, and could not be used to inform post-recurrence TPs. Therefore, the model assumed no sustained therapeutic benefit of adjuvant treatments after recurrence, potentially resulting in conservative estimates of incremental QALYs versus observation. This assumption should be re-examined as mature DMFS and OS data become available from KEYNOTE-054.

Finally, this cost-effectiveness analysis focused on direct healthcare costs from a payer perspective; indirect costs related to work productivity and caregiving were not considered. Including indirect costs would be expected to reduce ICERs by offsetting the cost of pembrolizumab treatment.

\section{Conclusion}

Results of this study showed that pembrolizumab reduced costs and extended survival relative to active comparators for the adjuvant treatment of completely resected stage III melanoma, dominating ipilimumab in the overall population and dabrafenib + trametinib in the BRAF+ subgroup. Pembrolizumab was estimated to increase costs relative to routine observation, with sufficient incremental benefit to be considered cost-effective from a US health system perspective. These conclusions were robust across a range of input values and assumptions. Future research is nevertheless warranted to validate these results against long-term survival in KEYNOTE-054 and comparator trials.

Acknowledgements The authors would like to thank the following individuals for their contributions to the analyses of clinical and quality of life data from KEYNOTE-054 to populate the model: Ruifeng $\mathrm{Xu}$, Principal Scientist Outcomes Research; Qian Wang, Principal Scientist Statistical Programming; Thao Nguyen, Associate Director, 
Quantitative Sciences; and Harris Kampouris, Associate Director, Statistical Programming. The authors would also like to thank Qian Xia, Associate Director, Outcomes Research, for contributions to the analyses of Flatiron registry data, which was also used to populate the model.

Author Contributions All authors contributed substantially to the design of the economic evaluation, data analysis and interpretation, and manuscript development.

\section{Compliance with Ethical Standards}

Funding This study was supported by funding from Merck Sharp \& Dohme Corp., a subsidiary of Merck \& Co., Inc., Kenilworth, NJ, USA.

Conflict of interest Arielle G. Bensimon, Zheng-Yi Zhou, Madeline Jenkins, Yan Song, Wei Gao, and James Signorovitch are employees of Analysis Group, Inc., which received consultancy fees from Merck and Co. in connection with this study. Clemens Krepler, Emilie Scherrer, Jingshu Wang, and Raquel Aguiar-Ibáñez are employees of Merck Sharp \& Dohme Corp., a subsidiary of Merck \& Co., Inc., Kenilworth, NJ, USA.

Open Access This article is licensed under a Creative Commons Attribution-NonCommercial 4.0 International License, which permits any non-commercial use, sharing, adaptation, distribution and reproduction in any medium or format, as long as you give appropriate credit to the original author(s) and the source, provide a link to the Creative Commons licence, and indicate if changes were made. The images or other third party material in this article are included in the article's Creative Commons licence, unless indicated otherwise in a credit line to the material. If material is not included in the article's Creative Commons licence and your intended use is not permitted by statutory regulation or exceeds the permitted use, you will need to obtain permission directly from the copyright holder.To view a copy of this licence, visit http://creativecommons.org/licenses/by-nc/4.0/.

\section{References}

1. Siegel RL, Miller KD, Jemal A. Cancer statistics, 2018. CA Cancer J Clin. 2018;68(1):7-30.

2. National Cancer Institute Surveillance Epidemiology and End Results Program. Cancer Stat Facts: Melanoma of the Skin 2018. https://seer.cancer.gov/statfacts/html/melan.html. Accessed 19 Mar 2019.

3. Balch CM, Gershenwald JE, Soong SJ, et al. Final version of 2009 AJCC melanoma staging and classification. J Clin Oncol. 2009;27(36):6199-206.

4. Garbe C, Peris K, Hauschild A, et al. Diagnosis and treatment of melanoma. European consensus-based interdisciplinary guideline-Update 2016. Eur J Cancer (Oxford, England: 1990). 2016;63:201-17.

5. National Comprehensive Cancer Network. Melanoma. NCCN clinical practice guidelines in oncology. Version 2.2019. https:// www.nccn.org/professionals/physician_gls/pdf/cutaneous_melan oma.pdf. Accessed 18 Aug 2019.

6. Mayo Clinic. Melanoma diagnosis and treatment 2016. https:// www.mayoclinic.org/diseases-conditions/melanoma/diagnosistreatment/drc-20374888. Accessed 31 Oct 2018.

7. Kudchadkar RR, Michielin O, van Akkooi ACJ. Practice-changing developments in stage III melanoma: surgery, adjuvant targeted therapy, and immunotherapy. Am Soc Clin Oncol Educ Book. 2018;38:759-62.
8. Tarhini AA, Lorigan P, Leachman S. Operable melanoma: screening, prognostication, and adjuvant and neoadjuvant therapy. Am Soc Clin Oncol Educ Book. 2017;37:651-60.

9. Sondak VK, Wolfe JA. Adjuvant therapy for melanoma. Curr Opin Oncol. 1997;9(7):189-204.

10. Herndon TM, Demko SG, Jiang X, et al. US Food and Drug Administration Approval: peginterferon-alfa- $2 b$ for the adjuvant treatment of patients with melanoma. Oncologist. 2012;17(10):1323-8.

11. Kirkwood JM, Ibrahim JG, Sondak VK, et al. High- and low-dose interferon alfa-2b in high-risk melanoma: first analysis of intergroup trial E1690/S9111/C9190. J Clin Oncol. 2000;18:2444-588.

12. Atkins MB, Kunkel L, Sznol M, et al. High-dose recombinant interleukin-2 therapy in patients with metastatic melanoma: longterm survival update. Cancer J Sci Am. 2000;6(Suppl 1):S11-S14.

13. Schuchter LM. Adjuvant melanoma therapy-head-spinning progress. N Engl J Med. 2017;377(19):1888-900.

14. Eggermont AMM, et al. Adjuvant ipilimumab versus placebo after complete resection of high-risk stage III melanoma (EORTC 18071): a randomised, double-blind, phase 3 trial. Lancet Oncol. 2015;16(5):522-30.

15. Eggermont AMM. Prolonged survival in stage III melanoma with ipilimumab adjuvant therapy. N Engl J Med. 2016;375:1845-55.

16. Weber J, Mandala M, Del Vecchio M, et al. Adjuvant nivolumab versus ipilimumab in resected stage III or IV melanoma. N Engl J Med. 2017;377(19):1824-35.

17. US Food and Drug Administration. FDA grants regular approval to nivolumab for adjuvant treatment of melanoma 2017. https:// www.fda.gov/drugs/informationondrugs/approveddrugs/ucm59 0004.htm. Accessed 15 Mar 2019.

18. Long GV, Hauschild A, Santinami M, et al. Adjuvant dabrafenib plus trametinib in stage III BRAF-mutated melanoma. N Engl J Med. 2017;377:1813-23.

19. US Food and Drug Administration. FDA approves dabrafenib plus trametinib for adjuvant treatment of melanoma with BRAF V600E or V600K mutations 2018. https://www.fda.gov/drugs/ informationondrugs/approveddrugs/ucm606165.htm. Accessed 15 Mar 2019.

20. US Food and Drug Administration. FDA approves pembrolizumab for adjuvant treatment of melanoma 2019. https://www.fda.gov/ Drugs/InformationOnDrugs/ApprovedDrugs/ucm631565.htm. Accessed 15 Mar 2019.

21. Eggermont AMM, Blank CU, Mandala M, et al. Adjuvant pembrolizumab versus placebo in resected stage III melanoma. $\mathrm{N}$ Engl J Med. 2018;378(19):1789-801.

22. Lorenzi M, Arndorfer S, Aguiar-Ibañez R, et al. An indirect treatment comparison of the efficacy of pembrolizumab versus competing regimens for the adjuvant treatment of stage III melanoma. J Drug Assess. 2019;8(1):135-45.

23. Sanders GD, Neumann PJ, Basu A, et al. Recommendations for conduct, methodological practices, and reporting of cost-effectiveness analyses: second panel on cost-effectiveness in health and medicine. JAMA. 2016;316(10):1093-103.

24. Bureau of Labor Statistics. Consumer price index: Medical care commodities in U.S. city average, all urban consumers, not seasonally adjusted. https://beta.bls.gov/dataViewer/view/timeseries /CUUR0000SAM1. Accessed 10 Apr 2020.

25. Siebert U, Alagoz O, Bayoumi AM, et al. State-transition modeling: a report of the ISPOR-SMDM Modeling Good Research Practices Task Force-3. Med Decis Making. 2012;32(5):690-700.

26. Bensimon AG, Zhou ZY, Jenkins M, et al. Cost-effectiveness of pembrolizumab for the adjuvant treatment of resected highrisk stage III melanoma in the United States. J Med Econ. 2019;22:981-93.

27. National Institute for Health and Care Excellence. Pembrolizumab for adjuvant treatment of resected melanoma with high risk of 
recurrence: Technology appraisal guidance [TA553] 2018. https ://www.nice.org.uk/guidance/ta553. Accessed 1 July 2019.

28. Williams C, Lewsey JD, Briggs AH, et al. Cost-effectiveness analysis in $\mathrm{R}$ using a multi-state modeling survival analysis framework: a tutorial. Med Decis Making. 2017;37(4):340-52.

29. Williams C, Lewsey JD, Mackay DF, et al. Estimation of survival probabilities for use in cost-effectiveness analyses: a comparison of a multi-state modeling survival analysis approach with partitioned survival and markov decision-analytic modeling. Med Decis Making. 2017;37(4):427-39.

30. National Institute for Health and Care Excellence. DSU Technical Support Document 19: Partitioned survival analysis for decision modelling in health care: a critical review 2017. https://niced su.org.uk/technical-support-documents/partitioned-survival-analy sis-tsd/. Accessed 2 Apr 2020.

31. Putter H, Fiocco M, Geskus RB. Tutorial in biostatistics: competing risks and multi-state models. Stat Med. 2007;26(11):2389-430.

32. Arias E, Heronm M, Xu J. United States life tables, 2014. Natl Vital Stat Rep. 2017;66:1-64.

33. R Core Team. R: A language and environment for statistical computing. R Foundation for Statistical Computing, Vienna, Austria. https://www.R-project.org/. Accessed 10 Mar 2019

34. National Institute for Health and Care Excellence. DSU Technical Support Document 14: Survival analysis for economic evaluations alongside clinical trials - extrapolation with patient-level data 2013. https://nicedsu.org.uk/wp-content/uploads/2016/03/ NICE-DSU-TSD-Survival-analysis.updated-March-2013.v2.pdf. Accessed 2 Apr 2020.

35. Flatiron Health. Flatiron database 2018. https://www.flatiron.com/ real-world-evidence. Accessed 2 Dec 2018.

36. Schachter J, Ribas A, Long GV, et al. Pembrolizumab versus ipilimumab for advanced melanoma: final overall survival results of a multicentre, randomised, open-label phase 3 study (KEYNOTE-006). Lancet. 2017;390(10105):1853-62.

37. Robert C, Karaszewska B, Schachter J, et al. Improved overall survival in melanoma with combined dabrafenib and trametinib. N Engl J Med. 2015;372(1):30-9.

38. Robert C, Long GV, Brady B, et al. Nivolumab in previously untreated melanoma without BRAF mutation. N Engl J Med. 2015;372(4):320-30.

39. Larkin J, Hodi FS, Wolchok JD. Combined nivolumab and ipilimumab or monotherapy in untreated melanoma. N Engl J Med. 2015;373(13):1270-1.

40. Hodi FS, Chesney J, Pavlick AC, et al. Combined nivolumab and ipilimumab versus ipilimumab alone in patients with advanced melanoma: 2-year overall survival outcomes in a multicentre, randomised, controlled, phase 2 trial. Lancet Oncol. 2016;17(11):1558-68

41. Ascierto PA, McArthur GA, Dreno B, et al. Cobimetinib combined with vemurafenib in advanced BRAF(V600)-mutant melanoma (coBRIM): updated efficacy results from a randomised, double-blind, phase 3 trial. Lancet Oncol. 2016;17(9):1248-60.

42. McArthur GACP, Robert C, et al. Safety and efficacy of vemurafenib in BRAFV600E and BRAFV600K mutation-positive melanoma (BRIM-3): extended follow-up of a phase 3, randomised, open-label study. Lancet Oncol. 2014;15:323-32.

43. Long GV, Stroyakovskiy D, Gogas H, et al. Dabrafenib and trametinib versus dabrafenib and placebo for Val600 BRAFmutant melanoma: a multicentre, double-blind, phase 3 randomised controlled trial. Lancet. 2015;386(9992):444-51.

44. Shaw JW, Johnson JA, Coons SJ. US valuation of the EQ-5D health states: development and testing of the D1 valuation model. Med Care. 2005;43(3):203-20.
45. Beusterien KMSS, Kotapati S, et al. Societal preference values for advanced melanoma health states in the United Kingdom and Australia. Br J Cancer. 2009;101:387.

46. Middleton MR, Atkins MB, Amos K, et al. Societal preferences for adjuvant melanoma health states: UK and Australia. BMC Cancer. 2017;17(1):689.

47. U.S. Department of Veterans Affairs. Pharmaceutical pricing data. https://www.va.gov/opal/nac/fss/pharmPrices.asp. Accessed $5 \mathrm{Apr}$ 2020.

48. Bristol-Myers Squibb Company. Yervoy (ipilimumab): prescribing information. 2018. https://packageinserts.bms.com/pi/ pi_yervoy.pdf. Accessed 10 Mar 2019.

49. Merck \& Co. Inc., Kenilworth, NJ, USA. Keytruda (pembrolizumab): Prescribing information. 2018. https://www.merck.com/ product/usa/pi_circulars/k/keytruda/keytruda_pi.pdf. Accessed 10 Mar 2019.

50. Centers for Medicare \& Medicaid Services. Hospital Outpatient Prospective Payment System. Jan 2020. https://www.cms.gov/ medicaremedicare-fee-service-paymenthospitaloutpatientppsad dendum-and-addendum-b-updates/january-2020-0. Accessed 14 Apr 2020.

51. Bristol-Myers Squibb Company. Opdivo (nivolumab): prescribing information. 2018. https://packageinserts.bms.com/pi/pi_opdiv o.pdf. Accessed 10 Mar 2019.

52. Genentech. Cotellic (cobimetinib): prescribing information. 2015 https://www.gene.com/download/pdf/cotellic_prescribing.pdf. Accessed 10 Mar 2019

53. Genentech. Zelboraf (vemurafenib): prescribing information. 2017. https://www.gene.com/download/pdf/zelboraf_prescribin g.pdf. Accessed 10 Mar 2019

54. Novartis Pharmaceuticals Corporation. Tafinlar (dabrafenib): prescribing information. 2018. https://www.pharma.us.novartis.com/ sites/www.pharma.us.novartis.com/files/tafinlar.pdf. Accessed 10 Mar 2019.

55. Novartis Pharmaceuticals Corporation. Mekinist (trametinib): prescribing information. 2018. https://www.pharma.us.novartis.com/ sites/www.pharma.us.novartis.com/files/mekinist.pdf. Accessed 10 Mar 2019.

56. National Institute for Health and Care Excellence. Ipilimumab for previously untreated advanced (unresectable or metastatic) melanoma [TA319] 2014. https://www.nice.org.uk/guidance/ta319. Accessed 10 Mar 2019.

57. National Institute for Health and Care Excellence. Pembrolizumab for treating advanced melanoma after disease progression with ipilimumab [TA357] 2017. https://www.nice.org.uk/guidance/ ta357. Accessed 10 Mar 2019.

58. Centers for Medicare \& Medicaid Services. Acute Inpatient Prospective Payment System. https://www.cms.gov/Medicare/Medic are-Fee-for-Service-Payment/AcuteInpatientPPS/FY2018-IPPSFinal-Rule-Home-Page.html. Accessed 15 Sep 2019.

59. Rashid N, Koh HA, Baca HC, et al. Economic burden related to chemotherapy-related adverse events in patients with metastatic breast cancer in an integrated health care system. Breast Cancer (Dove Med Press). 2016;8:173-81.

60. Wong W, Yim YM, Kim A, et al. Assessment of costs associated with adverse events in patients with cancer. PLoS ONE. 2018;13(4):e0196007.

61. Barzey V, Atkins MB, Garrison LP, et al. Ipilimumab in 2nd line treatment of patients with advanced melanoma: a cost-effectiveness analysis. J Med Econ. 2013;16(2):202-12.

62. Zhang Y, Le TK, Shaw JW, et al. Retrospective analysis of drug utilization, health care resource use, and costs associated with IFN therapy for adjuvant treatment of malignant melanoma. ClinicoEcon Outcomes Res CEOR. 2015;7:397-407.

63. Merck Sharp \& Dohme Corp., Whitehouse Station, NJ, USA. Adjuvant immunotherapy with anti-PD-1 monoclonal antibody 
Pembrolizumab (MK-3475) versus placebo after complete resection of high-risk Stage III melanoma: A randomized, double-blind Phase 3 trial of the EORTC Melanoma Group: Clinical study report. Data cutoff date: 2 Oct 2017.

64. Seidler AM, Pennie ML, Veledar E, et al. Economic burden of melanoma in the elderly population: population-based analysis of the Surveillance, Epidemiology, and End Results (SEER)—Medicare data. Arch Dermatol. 2010;146(3):249-56.

65. Tarhini A, Corman SL, Rao S, et al. Healthcare resource utilization and associated costs in patients with advanced melanoma receiving first-line ipilimumab. J Cancer Ther. 2015;6:833-40.

66. Chastek B, Harley C, Kallich J, et al. Health care costs for patients with cancer at the end of life. J Oncol Pract. 2012;8(6):75s-80s.

67. Eddy DM, Hollingworth W, Caro JJ, et al. Model transparency and validation: a report of the ISPOR-SMDM Modeling Good Research Practices Task Force-7. Med Decis Making. 2012;32(5):733-43.

68. Institute for Clinical and Economic Review. Overview of the ICER value assessment framework and update for 2017-2019. https:// icer-review.org/wp-content/uploads/2017/06/ICER-value-asses sment-framework-Updated-050818.pdf. Accessed 1 Feb 2018.

69. Institute for Clinical and Economic Review. ICER's reference case for economic evaluations: Principles and rationale. 16 July 2018.
https://icer-review.org/wp-content/uploads/2018/07/ICER_Refer ence_Case_July-2018.pdf. Accessed 9 Feb 2019.

70. Suciu S, Eggermont AMM, Lorigan P, et al. Relapse-free survival as a surrogate for overall survival in the evaluation of stage II-III melanoma adjuvant therapy. J Natl Cancer Inst. 2018;110(1):87-96.

71. National Institute for Health and Care Excellence. Nivolumab for adjuvant treatment of completely resected melanoma with lymph node involvement or metastatic disease [TA558] 2019. https:// www.nice.org.uk/guidance/ta558. Accessed 12 Oct 2019.

72. National Institute for Health and Care Excellence. Dabrafenib with trametinib for adjuvant treatment of resected BRAF V600 mutation-positive melanoma [TA544] 2018. https://www.nice.org. uk/guidance/ta544. Accessed 1 July 2019.

73. National Institute for Health and Care Excellence. Appraisal consultation document: Nivolumab for adjuvant treatment of completely resected melanoma with lymph node involvement or metastatic disease. August 2018. https://www.nice.org.uk/guida nce/gid-ta10286/documents/appraisal-consultation-document. Accessed 12 Oct 2019. 